\title{
Educación no formal e inserción laboral de la juventud
}

\author{
Non formal education and youth labour insertion
}

\author{
Ana Vázquez Rodríguez*, Jesús García Álvarez*, Alexandre Sotelino Losada** \\ *GI ESCULCA (Rede de Investigación RIES) - Universidad de Santiago de Compostela \\ **GI ESCULCA (Rede de Investigación RIES) - Universidad de Vigo
}

\begin{abstract}
Resumen
En este trabajo pretendemos analizar el alcance estratégico de la formación del capital humano y social que implica la participación en actividades de educación no formal, considerando las aportaciones que vinculan tal participación con indicadores de empleabilidad en la sociedad actual. Con esta finalidad, nos centramos en el análisis pormenorizado del desarrollo de competencias transversales que suponen la participación en actividades de educación no formal estimándolas claves en la mejora de la inserción laboral y social del colectivo juvenil en la Comunidad Autónoma de Galicia, un contexto señalado en el reconocimiento y acreditación de los aprendizajes no formales.

Palabras clave: educación no formal, oportunidades de empleo, competencia, empleo juvenil.
\end{abstract}

\begin{abstract}
The aim of this project is the analysis of the strategic scope of the formation of human and social capital that involves the participation in non-formal education, considering the contributions that such participation links with the employability improvement in current society. For this purpose, we focus on the detailed analysis of the development of transversal competences that involve the participation in non-formal activities', considering them as important to improve the employability and social inclusion of the youth in Galicia, a context indicated in the recognition and accreditation of non-formal learning. Keywords: non-formal education, employment opportunities, competence, youth employment.
\end{abstract}

\section{Introducción}

La relevancia de la educación no formal encuentra su sentido en diversas aportaciones desde el marco europeo, que vinculan su notable importancia en su contribución al aprendizaje permanente y en su contienda en relación al abandono escolar prematuro (Comisión de las Comunidades Europeas, 2009).

Asimismo, teniendo en cuenta indicadores de la situación actual, el reconocimiento de los aprendizajes no formales alberga una gran significación en los actuales procesos de crisis económica, una coyuntura que remarca sus graves consecuencias a través de altas tasas de desempleo de la población juvenil.

En este sentido, si tomamos como referencia la Comunidad Gallega entre los años 2009 y el primer trimestre del año 2015, podemos dilucidar un escenario bastante desalentador en relación a las tasas de ocupación y desempleo de la población juvenil (véase Figura 1):

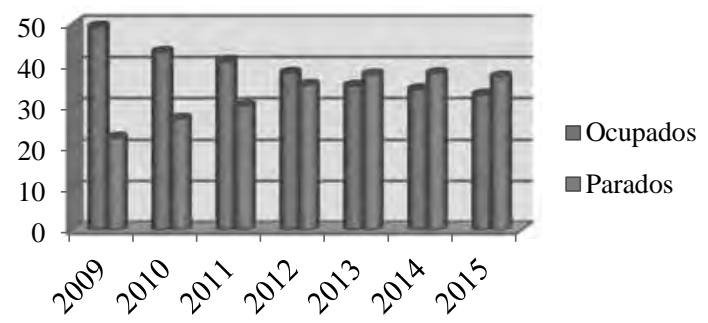

Figura 1. Tasa de ocupación y de paro del colectivo juvenil gallego, en porcentaje (2009-2015). Elaboración propia a partir del IGE (2015).

Consecuentemente, tal y como puede advertirse, es a partir del año 2013, cuando la tasa de parados jóvenes empieza a superar la de ocupados en la Comunidad Gallega. Igualmente, podemos esclarecer cómo desde el año 2009 la tasa de parados empieza a incrementarse gradualmente, existiendo un pico en el año 2014 (situado en un 38\%) y disminuyendo casi imperceptiblemente al año siguiente.

Desde esta perspectiva, se hace emergente la creación de mecanismos, iniciativas y programas que logren incidir en una mejora palpable de la inserción laboral juvenil en un panorama en el que los requisitos por parte del mercado laboral son cada vez más estrictos en términos de cualificaciones y competencias. Una serie de requerimientos por parte de los empleadores que incluyen el desarrollo de competencias individuales tanto actuales como potenciales de la persona, en tanto que éstas representan una baza importantísima para la empresa (Lévy-Leboyer, 2003).

En este sentido, el establecimiento de un marco común de validación y certificación de los aprendizajes no formales cumple una labor esencial en el reconocimiento del compendio de competencias transversales adquiridas a través de dichos procesos, concediéndoles una mayor visibilidad para los potenciales empleadores.

Un propósito fundamental del que depende en buena medida la inserción laboral de nuestros jóvenes y que el Consejo Europeo (2012) ha querido ratificar a través de una medida pionera en el marco europeo, la Recomendación sobre la validación del aprendizaje no formal e informal. Concretamente, desde este escrito comunitario se insta a los Estados Miembros de la Unión Europea, de manera precedente, a desarrollar acciones específicas para la certificación de determinadas competencias a través de los aprendizajes adquiridos mediante vías no formales de formación, estimándolos clave en la mejora del empleo y la movilidad. 
Desde este punto de vista, el desarrollo de competencias transversales adquiridas a través de procesos como la participación en organizaciones juveniles o el voluntariado dentro de la educación no formal, se configura como una pieza clave a la hora de acceder a un puesto laboral. Una condición que, por ende, atribuiría un valor añadido a las cualificaciones que son obtenidas por la educación formal, cuyos procesos están más dirigidos hacia la adquisición de competencias específicas de cada profesión.

Aun así, si tomamos como referencia el marco europeo observamos cómo el quehacer del reconocimiento de la educación no formal es una tarea ardua y compleja, tanto por la minusvalorización presente de los aprendizajes no formales, como por la necesidad de establecer puntos de acuerdo de cómo han de desarrollarse los procesos de validación y certificación y con qué metodologías. Además, tal y como señalan Damesin, Fayolle, y Fleury (2012), la existencia de un marco nacional no tiene por qué ser sinónimo de resultados cualitativos importantes, ni de efectividad de prácticas de validación.

Así y todo, la esencialidad de este trabajo radica en la trascendencia de hacer efectivo el derecho al trabajo del colectivo juvenil, un propósito esencial para el que consideramos básico la validación y acreditación de los aprendizajes no formales, así como la necesidad de establecer metodologías fiables destinadas a la identificación y validación sistemática de tales aprendizajes; dos cuestiones que mejoran, incontestablemente, su reconocimiento por parte de la sociedad (Bjornavold, 1997).

\section{Método}

Para el siguiente estudio hemos empleado, sustancialmente, una metodología de tipo cualitativo. Congruentemente, desde los principios vertebradores de esta metodología de investigación educativa, realizamos una minuciosa revisión bibliográfica de nuestro objeto de estudio situado en el ámbito de la educación no formal y la inserción laboral de la juventud.

Además, con la finalidad de profundizar en nuestro tema de investigación, hemos realizado un análisis exhaustivo de publicaciones no solo nacionales, sino también internacionales. A este respecto, hemos valorado e interpretado en este trabajo, las contribuciones más representativas en relación a nuestro objeto de estudio, esto es, informes internacionales, estudios en perspectiva educativa $y$, en general, sopesando cualquier aportación que pudiera ser de relevancia para esta investigación documental.

Igualmente, dentro del marco investigador que nos concierne, queremos destacar la importancia de este estudio ya que desde éste entroncamos con una nueva perspectiva que reafirma a los procesos educativos no formales como adecuados para la mejora de la inserción laboral de la juventud, rompiendo coyunturalmente con la relación tradicional exclusiva entre educación formal y empleabilidad.

\section{Resultados}

Como es vigente, partimos de una realidad que condiciona el cómo y el cuándo se produce la inserción laboral de los jóvenes en Galicia, confluyendo una serie de determinantes que inician o revocan la posibilidad de este colectivo de conseguir un trabajo adecuado a su nivel de formación y a sus aspiraciones profesionales. Un contexto, para el que consideramos indispensable la elaboración de un análisis de las demandas derivadas del entorno empresarial, resaltando su importancia en torno a las competencias y cualificaciones que deben poseer los jóvenes $\mathrm{y}$, su consideración acerca de cuáles son las más solicitadas para desempeñar un trabajo adaptado a las circunstancias actuales.

En primera instancia, aproximándonos al término de competencia, hacemos alusión a su origen en la conformidad del sistema educativo y su estimación en el mercado laboral en relación a la mejora de la empleabilidad del colectivo juvenil. Un concepto que, además, debemos distinguir de la noción de cualificación profesional ya que, aunque normalmente son confundidos, son términos completamente divergentes.

A este respecto, debemos concretar que existe una diversidad emergente de concepciones en relación al concepto de competencia y, como resultado, es imposible imputarle una teoría coherente y una definición capaz de acomodarse y reconciliar las múltiples maneras en que éste es usado (Delamare y Winterton, 2005). No obstante, nos centramos en la definición de competencia recogida por Fernández Salinero (2006, p. 137) que las detalla como "una compleja estructura de atributos necesarios para el desempeño de situaciones diversas, donde se combinan conocimientos (tácitos y explícitos), habilidades, actitudes y valores, con tareas que se tienen que desempeñar en determinadas situaciones”.

De esta manera, las competencias han de ser claramente distinguidas de las cualificaciones profesionales, dos conceptos que son complementarios y, a la vez, condicionantes de la inserción laboral de la juventud. Concretamente, las cualificaciones profesionales son las acreditadas por un título de formación, un certificado de competencia y/o una experiencia profesional, y a efectos del Marco Europeo de Cualificaciones (Comisión Europea, 2009), son el resultado formal de un proceso de evaluación y validación que indica cuando un individuo ha superado un nivel determinado.

A este respecto, entendemos que la cualificación es el resultado que valida definitoriamente, en nuestro caso, la adquisición de competencias estipuladas desde los organismos y leyes pertinentes de cada país.

En este contexto, afirmamos que las competencias y su transformación en las cualificaciones correspondientes en cada caso, mejoran la inserción laboral de la juventud en su dimensión de promoción de la empleabilidad (González y Wagenaar, 2003), este último término entendido como una serie de logros -habilidades, conocimientos y atributos personales- que hacen que las personas aumenten su probabilidad de conseguir un empleo y tener éxito en sus ocupaciones, con beneficios para sí mismos, la fuerza de trabajo, la comunidad y la economía (Yorke, 2004). 
En este sentido, es indiscutible el papel esencial que juegan las competencias en el desarrollo personal y profesional de todas las personas (McQuaid y Lindsay, 2005; Santos Rego, 2014). De tal manera, la validación de las competencias adquiridas, emerge en nuestras realidades con un rol esencial en la mejora de la empleabilidad del colectivo juvenil, aunque esta dimensión siempre debe estar aunada al respaldo de políticas activas de empleo que mejoren las condiciones precarias en las que se encuentra el mercado laboral en la actualidad.

Aun así, es decisivamente reseñable el papel que las competencias genéricas o transversales desempeñan en la inserción laboral, ya que cada vez son más demandadas por parte de los empleadores, un hecho que ha impulsado a las empresas a elaborar sistemas de referencia de competencias que les ayuden a conocer sus recursos actuales y potenciales (Lévy-Levoyer, 2003).

Una contribución muy interesante que confirma la esencialidad del desarrollo de competencias en nuestras sociedades, es la registrada en uno de los últimos Foros de la Organización para la Cooperación y Desarrollo Económico (OCDE, 2014), en el que se debatía sobre las Habilidades para el progreso social y en el que se llega a concluir que, siendo las habilidades la moneda global del Siglo XXI, necesitamos incrementar nuestro pensamiento y considerar el invertir en un más amplio rango de habilidades, donde las sociales y emocionales sean tan importantes como las cognitivas.

En otras palabras, las posibilidades de inserción laboral de nuestros jóvenes no sólo vienen determinadas por la capacidad que los futuros trabajadores demuestren al adaptarse a las demandas laborales, sino también por la razón de que los empleadores puedan albergar una imagen completa y profunda del futuro trabajador a la hora de contratarlo (sus habilidades interpersonales, inquietudes emocionales...), cumpliendo la certificación de competencias un rol decisivo al respecto.

Como resultado, con el objeto de hacer un análisis pormenorizado de las competencias que se desarrollan a través de los procesos de aprendizaje no formal y de su valorización por parte de los empleadores, partimos de un informe reciente, pionero en Europa, denominado Estudio sobre el impacto de la educación no formal en la empleabilidad de la juventud (Souto, Ulicna, Schaepkens, y Bognar, 2012). En general, estableciendo relaciones entre las competencias transversales adquiridas por los jóvenes a través de procesos de educación no formal y las demandadas por el mercado laboral, ofrecemos la representación del nivel de desarrollo de competencias por parte del colectivo juvenil (en una escala de o a 5) según su nivel formativo (véase Figura 2).

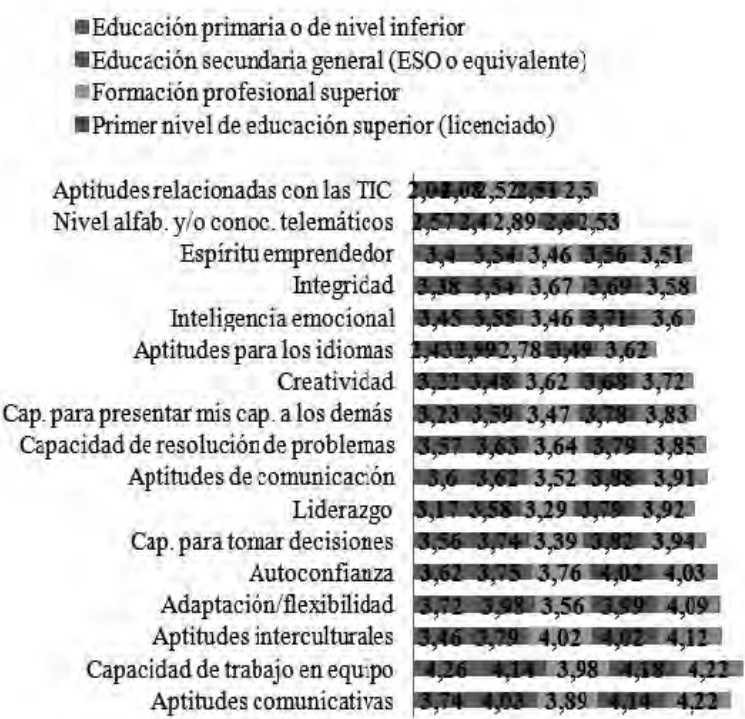

Figura 2. Nivel individual de desarrollo de competencias en organizaciones juveniles por nivel de logros educativos. Adaptado de Souto et al. (2012, p. 51).

En conjunto, tomando como referencia los datos representativos del colectivo juvenil, a través de este estudio podemos señalar dos aspectos diferenciados: por un lado, que los jóvenes con más cualificación, consideran que tienen un mayor desarrollo de competencias transversales mediante su participación en actividades de educación no formal; y, por otro, que este colectivo tiene una muy buena estimación del desarrollo de competencias promovidas desde su participación en estas actividades ya que, en su mayoría, se sitúan por valores encima de 3 .

En esta misma línea, a partir de esta investigación se concreta que los jóvenes valoran considerablemente el grado de influencia de su experiencia en organizaciones juveniles a la hora de conseguir un trabajo, manifestando gran parte de ellos (81\%) que les ayudan considerablemente en sus oportunidades de inserción laboral. Igualmente, junto con el desarrollo de competencias y el aumento de la empleabilidad expresado por el colectivo juvenil, uno de los aspectos que se señalan es la posibilidad de ver aumentada su propia inserción laboral gracias a la red de contactos que logra erigirse a través de su implicación en dichos procesos.

Una afirmación que es reflejada en la opinión de los jóvenes encuestados que afirman que los contactos obtenidos le hicieron darse cuenta de las oportunidades de empleo (36\%), que les han ayudado en cuestiones de empleo (21\%) o que sí son útiles pero que de momento no les han influenciado a la hora de encontrar un trabajo (32\%). 
Asimismo, desde el estudio de Souto et al. (2012), se confirma nuestra hipótesis inicial de que la experiencia en organizaciones juveniles aporta una información valiosa a los empleadores sobre las motivaciones que tiene la persona candidata y su talento potencial para encajar en la organización. Un aspecto que, afirman, depende del nivel de formalización de la participación en organizaciones juveniles, siendo el empleo de certificados y el registro de documentos muy importantes en este sentido.

A este respecto, entendiendo la validación y certificación de las competencias desarrolladas mediante aprendizajes no formales, independientemente del modo de adquisición, como un derecho individual que debe ser accesible en la práctica a todas las personas (Damesin et al., 2012), resaltamos convenientemente la envergadura del Certificado de Experiencias de Educación No Formal en materia de juventud (DOG, 25/3/2015). Un certificado, promovido desde la administración gallega, que trata de concederle más notoriedad a los aprendizajes adquiridos mediante cualquier vía de formación dentro del marco del aprendizaje permanente (Consejo Europeo, 2012) y de asegurar un acceso más efectivo a la información y a otros recursos relacionados con el empleo (Santos Rego, 2014).

De igual manera, desde el contexto gallego, debemos señalar una serie de programas destinados a contribuir al desarrollo personal de los jóvenes, cuyo propósito final es el enriquecer su currículo vital y favorecer su participación activa en la sociedad. Estamos hablando de un conjunto de programas, entre los que se incluyen Iniciativa Xove, Erasmus +, Juventud en acción, La Juventud en el mundo, Campos de trabajo y Galeuropa, reconocidos oportunamente para su certificación (DOG, $25 / 3 / 2015$ ), y que consiguen asignarle una considerable visibilidad a los procesos de aprendizaje no formal y a las competencias adquiridas a través de los mismos. Asimismo, desde nuestra opinión, estos programas además de vincular a la juventud en sus contextos, fomentan la construcción de una identidad personal dinámica, participativa, sensible a las diferencias y comprometida con la dignidad humana (Gonzálvez y García López, 2013). Precisamente, estos programas sirven a propósitos diferenciados que se vuelven cada vez más apremiantes en nuestras realidades como son el voluntariado, la movilidad, la solidaridad y, dentro del ámbito laboral, el fomento de la empleabilidad y el emprendimiento.

Ahora bien, tras un análisis de cada uno de ellos y de su posible repercusión en la inserción laboral de los jóvenes en Galicia, nos centramos en el Programa Iniciativa Xove en tanto que, sopesamos, es un ejemplo representativo de cómo puede potenciarse el liderazgo social de la juventud. Así pues, destacamos la labor que concierne a los fines de este programa en su dimensión de desarrollo de empleabilidad de los jóvenes gallegos, convirtiéndose meritoriamente en una verdadera escuela de ciudadanía activa que potencia el talento de la juventud gallega a través del financiamiento de proyectos dirigidos y gestionados por ellos mismos.
En este sentido, el objetivo general del Programa Iniciativa Xove es la concesión de ayudas para la puesta en marcha de iniciativas juveniles, entendidas como proyectos liderados por la juventud gallega, en los que pueden participar activamente a través de su planificación y realización. Igualmente, recogiendo todas las cualidades ligadas a este programa que inciden de manera directa en la inserción laboral de la juventud, debemos resaltar una de sus características más sobresalientes y es que sus beneficiarios no son únicamente las organizaciones juveniles sin ánimo de lucro, sino también los aclamados grupos informales, es decir, agrupaciones no institucionales de jóvenes que desean desarrollar una iniciativa juvenil y que cumplan con los requisitos establecidos en las distintas convocatorias publicadas por el Diario Oficial de Galicia.

A este respecto, debemos resaltar la trascendencia que concierne a la modalidad recogida en este programa organizada mediante grupos informales, ya que abre el concepto de participación hacia una perspectiva más amplia, rompiendo con las estructuras clásicas en las que se dependía exclusivamente de una organización juvenil para la implicación en una determinada problemática social.

Como resultado, los participantes mediante grupos informales pueden adquirir, de manera más preeminente, competencias sustantivas como la creatividad, el trabajo en equipo, el liderazgo o la autonomía, distinguiéndolos de otros jóvenes de su edad e implicándolos en un proyecto que realiza sus expectativas profesionales, escasamente satisfechas desde el ámbito laboral.

En general, consideramos que este programa exalta, de manera encomiable, la transición entre formación y empleo tratando de rasgar los moldes en los que las políticas de la juventud desarrollaban prioritariamente sus acciones e inaugurando un camino en el que los jóvenes sean quienes de tener voz, haciendo realidad sus inmanentes expectativas personales y profesionales. Un programa que, en definitiva, acompaña a los jóvenes en su desarrollo personal y social, y les ayuda en la adquisición de competencias fundamentales en la ardua tarea de acceder a un empleo en tiempos de extraordinaria incertidumbre.

\section{Discusión}

A lo largo de este análisis no hemos querido más que demostrar la calidad y pertinencia de la educación no formal en el ámbito pedagógico como un proceso educativo que alza, en plenitud, el desarrollo social y profesional de todas las personas que apuestan por el propósito capital de aprender a lo largo de toda la vida.

Nuestra concepción se emplaza, por tanto, en la superación de la visión tradicional que supedita las exigencias educativas únicamente a los procesos formales, íntimamente regulados e institucionalizados, hacia un pensamiento que revela las extensas posibilidades subyacentes a la educación no formal, cuyos ejes de actuación se aúnan a la flexibilización de intereses y la participación ciudadana. Siendo así, un paisaje educativo que abre su mirada a todos los cauces que se derivan de la educación, en una época donde las demandas y expectativas sociales se bifurcan en senderos 
interminables. No obstante, valorar la idoneidad de la educación no formal y su esencia dentro del aprendizaje permanente, supone discernirla en su tarea meritoria en el desarrollo de competencias transversales que se tornan fundamentales en la búsqueda de un empleo digno, adecuado a las aspiraciones personales y profesionales de todas las personas.

Así y todo, a lo largo de este trabajo hemos podido dilucidar cómo la Comunidad Autónoma de Galicia desempeña un rol trascendental en el reconocimiento y validación de los aprendizajes no formales, siendo una comunidad claramente proactiva en la certificación y disposición de iniciativas dentro de la educación no formal que den cabida a un reconocimiento más extenso dentro de la sociedad en su conjunto. Una inserción que, como vimos, está basada en el desarrollo de competencias transversales que pueden ser valoradas como vitales en la empleabilidad de la juventud, pero también dentro del ámbito social. A fin de cuentas, estamos refiriéndonos a una serie de competencias como las aptitudes comunicativas, la autoconfianza, la capacidad de trabajo en equipo, las aptitudes interculturales o la inteligencia emocional, que son adquiridas mediante la participación en organizaciones juveniles (Souto et al., 2012) y que actúan conforme al desarrollo de una competencia cívica que engrandece la dimensión de sociedad. Es, por tanto, elemental la participación de nuestros jóvenes en las organizaciones de la sociedad civil como un instrumento que les permita cumplir, de manera efectiva, sus intereses y proteger sus creencias (Youniss et al., 2002).

Sin embargo, es hoy más que nunca, en un mundo convulso y desigual, cuando se vuelve más necesaria la estrecha harmonía que supone colaborar en un objetivo común que lleve a la adquisición de competencias interculturales y sociales, volviendo más crítica a la juventud en la afanosa tarea de la lucha en contra de la exclusión social y de los conflictos que se erigen con fuerza en nuestros entornos. Unas competencias desarrolladas a través de procesos de educación no formal que, además, promueven la empleabilidad de la juventud en la persecución de hacer realidad su derecho al trabajo y, en definitiva, de hacer palpables sus máximas aspiraciones personales y profesionales.

\section{Referencias}

Bjornavold, J. (1997). La evaluación del aprendizaje no formal: calidad y limitaciones de las metodologías. Revista Europea de Formación Profesional, 12, 58-75.

Comisión de las Comunidades Europeas. (2009). Una estrategia de la UE para la juventud: inversión y capacitación. Un método abierto de coordinación renovado para abordar los desafíos y las oportunidades de los jóvenes. Recuperado de http://eur-lex.europa.eu/legal-content/ES/TXT/PDF/? uri=CELEX:52009DC0200\&from=ES

Comisión Europea. (2009). El Marco Europeo de Cualificaciones para el aprendizaje permanente (EQF-MEC). Bélgica: Oficina de Publicaciones Oficiales de las Comunidades Europeas.
Consejo Europeo. (2012). Recomendación del Consejo 20 de Diciembre de 2012 sobre la validación del aprendizaje no formal e informal. Recuperado de http://eur-lex.europa.eu/legal-content/ES/TXT/PDF/? uri=CELEX:32012H1222(01)\&from=ES

Damesin, R., Fayolle, J., y Fleury, N. (2012). Agentes, prácticas y desafíos del NFIL, así como su validación en Europa. Informe de la Confederación Europea de Sindicatos con el respaldo de la Comisión Europea. Recuperado de https://www.etuc.org/sites/www. etuc.org/files/ES_-_NFIL_Rapport_de_Synthese_FINA L.pdf

Delamare, F., y Winterton, J. (2005). What is competence?. Human Resource Development International, 8 (1), 27-46.

Fernández Salinero, C. (2006). Las competencias en el marco de la convergencia europea: Un nuevo concepto para el diseño de programas educativos. Encounters on Education, 7, 131-153.

González, J. y Wagenaar, R. (2003). Tuning Educational Structures in Europe. Informe Final. Bilbao: Universidad de Deusto.

Gonzálvez, V., y García López, R. (2013). Cosmopolitismo cívico: cooperación, educación y desarrollo. En M. A. Santos Rego (Ed.), Cosmopolitismo y educación. Aprender y trabajar en un mundo sin fronteras (pp. 33-48). Valencia: Brief Ediciones.

IGE. (2015). Poboación entre 16 e 29 anos por sexo, grupos de idade e relación coa actividade económica. Recuperado de http://www.ige.eu/igebdt/selector.jsp?COD=6516\&p axina $=001 \& c=0204023$

Lévy-Leboyer, C. (2003). Gestión de las competencias. Cómo analizarlas, cómo evaluarlas, cómo desarrollarlas. Barcelona: Gestión 2000.

McQuaid, R. W., y Lindsay, C. (2005). The concept of employability. Urban Studies, 42(2), 197-219.

OCDE. (2014). Foro de Políticas de Alto Nivel sobre "Habilidades para el progreso social”. Recuperado de http://www.oecd.org/centrodemexico/medios/forode politicasdealtonivelsobrehabilidadesparaelprogresosocia l.htm

Santos Rego, M. A. (2014). Fines de la Universidad y retos de la empleabilidad en la Educación Superior. Procedia-Social and Behavorial Sciences, 139, 16-25.

Souto, M., Ulicna, D., Schaepkens, L., y Bognar, V. (2012). Estudio sobre el impacto de la educación no formal en la empleabilidad de la juventud. Bruselas: Foro Europeo de la Juventud.

Yorke, M. (2004). Employability in higher education: what is it-what is not. York: Higher Education Academy.

Youniss, J., Bales, S., Christmas-Best, V., Diversi, M., McLaughlin, M., y Silbereisen, R. (2002). Youth Civic Engagement in the Twenty-First Century. Journal of Research on Adolescence, 12 (1), 121-148. 